\title{
A combinatorial proof of Postnikov's identity and a generalized enumeration of labeled trees
}

\author{
Seunghyun Seo* \\ Department of Mathematics \\ Brandeis University, Waltham, MA 02454, USA \\ shseo@brandeis.edu
}

Submitted: Sep 16, 2004; Accepted: Dec 16, 2004; Published: Jan 24, 2005

Mathematics Subject Classifications: 05A15, 05C05, 05C30

\begin{abstract}
In this paper, we give a simple combinatorial explanation of a formula of A. Postnikov relating bicolored rooted trees to bicolored binary trees. We also present generalized formulas for the number of labeled $k$-ary trees, rooted labeled trees, and labeled plane trees.
\end{abstract}

\section{Introduction}

In Stanley's 60th Birthday Conference, Postnikov [3, p. 21] showed the following identity:

$$
(n+1)^{n-1}=\sum_{\mathfrak{b}} \frac{n !}{2^{n}} \prod_{v \in V(\mathfrak{b})}\left(1+\frac{1}{h(v)}\right),
$$

where the sum is over unlabeled binary trees $\mathfrak{b}$ on $n$ vertices and $h(v)$ denotes the number of descendants of $v$ (including $v$ ). The figure below illustrates all five unlabeled binary trees on 3 vertices, with the value of $h(v)$ assigned to each vertex $v$. In this case, identity (1) says that $(3+1)^{2}=3+3+4+3+3$.
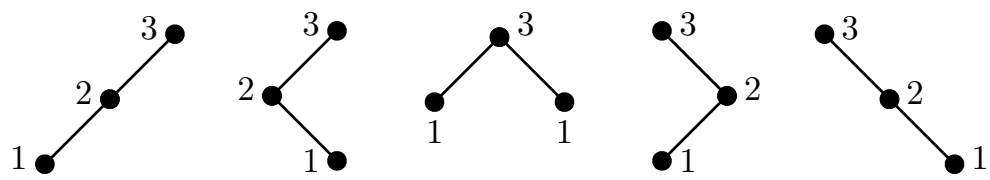

${ }^{*}$ Research supported by the Post-doctoral Fellowship Program of Korea Research Foundation (KRF). 
Postnikov derived this identity from the study of a combinatorial interpretation for mixed Eulerian numbers, which are coefficients of certain reparametrized volume polynomials which introduced in [3]. For more information, see [2, 3].

In the same talk, he also asked for a combinatorial proof of identity (1). Multiplying both sides of (1) by $2^{n}$ and expanding the product in the right-hand side yields

$$
2^{n}(n+1)^{n-1}=\sum_{\mathfrak{b}} n ! \sum_{\alpha \subseteq V(\mathfrak{b})} \prod_{v \in \alpha} \frac{1}{h(v)} .
$$

Let $\mathrm{LHS}_{n}$ (resp. RHS ) denote the left-hand (resp. right-hand) side of (2).

The aim of this paper is to find a combinatorial proof of (2). In Section 2 we construct the sets $\mathcal{F}_{n}^{\text {bi }}$ of labeled bicolored forests on $[n]$ and $\mathcal{D}_{n}$ of certain labeled bicolored binary trees, where the cardinalities equal $\mathrm{LHS}_{n}$ and $\mathrm{RHS}_{n}$, respectively. In Section 3 we give a bijection between $\mathcal{F}_{n}^{\text {bi }}$ and $\mathcal{D}_{n}$, which completes the bijective proof of (2). Finally, in Section 4 , we present generalized formulas for the number of labeled $k$-ary trees, rooted labeled trees, and labeled plane trees.

\section{Combinatorial objects for $\mathrm{LHS}_{n}$ and $\mathrm{RHS}_{n}$}

From now on, unless specified, we consider trees to be labeled and rooted.

A tree on $[n]:=\{1,2, \ldots, n\}$ is an acyclic connected graph on the vertex set $[n]$ such that one vertex, called the root, is distinguished. We denote by $\mathcal{T}_{n}$ the set of trees on $[n]$ and by $\mathcal{T}_{n, i}$ the set of trees on $[n]$ where vertex $i$ is the root. A forest is a graph such that every connected component is a tree. Let $\mathcal{F}_{n}$ denote the set of forests on $[n]$. There is a canonical bijection $\gamma: \mathcal{T}_{n+1, n+1} \rightarrow \mathcal{F}_{n}$ such that $\gamma(T)$ is the forest obtained from $T$ by removing the vertex $n+1$ and letting each neighbor of $n+1$ be a root. A graph is called bicolored if each vertex is colored with the color $\mathbf{b}$ (black) or $\mathbf{w}$ (white). We denote by $\mathcal{F}_{n}^{\text {bi }}$ the set of bicolored forests on $[n]$. From Cayley's formula [1] and the bijection $\gamma$, we have

$$
\left|\mathcal{F}_{n}\right|=\left|\mathcal{T}_{n+1, n+1}\right|=(n+1)^{n-1} \quad \text { and } \quad\left|\mathcal{F}_{n}^{\mathrm{bi}}\right|=2^{n} \cdot(n+1)^{n-1} .
$$

Thus $\mathrm{LHS}_{n}$ can be interpreted as the cardinality of $\mathcal{F}_{n}^{\text {bi }}$.

Let $F$ be a forest and let $i$ and $j$ be vertices of $F$. We say that $j$ is a descendant of $i$ if $i$ is contained in the path from $j$ to the root of the component containing $j$. In particular, if $i$ and $j$ are joined by an edge of $F$, then $j$ is called a child of $i$. Note that $i$ is also a descendant of $i$ itself. Let $S(F, i)$ be the induced subtree of $F$ on descendants of $i$, rooted at $i$. We call this tree the descendant subtree of $F$ rooted at $i$. A vertex $i$ is called proper if $i$ is the smallest vertex in $S(F, i)$; otherwise $i$ is called improper. Let $\operatorname{pv}(F)$ denote the the number of proper vertices in $F$.

A plane tree or ordered tree is a tree such that the children of each vertex are linearly ordered. We denote by $\mathcal{P}_{n}$ the set of plane trees on $[n]$ and by $\mathcal{P}_{n, i}$ the set of plane trees on $[n]$ where vertex $i$ is the root. Define a plane forest on $[n]$ to be a finite ordered sequence of non-empty plane trees $\left(P_{1}, \ldots, P_{m}\right)$ such that $[n]$ is the disjoint union of the 
sets $V\left(P_{r}\right), 1 \leq r \leq m$. We denote by $\mathcal{P F}_{n}$ the set of plane forests on $[n]$ and by $\mathcal{P F}_{n}^{\text {bi }}$ the set of bicolored plane forests on $[n]$. There is also a canonical bijection $\bar{\gamma}: \mathcal{P}_{n+1, n+1} \rightarrow \mathcal{P F}_{n}$ such that $\bar{\gamma}(P)=\left(S\left(P, j_{1}\right), \ldots, S\left(P, j_{m}\right)\right)$ where each vertex $j_{r}$ is the $r$ th child of $n+1$ in $P$. It is well-known that the number of unlabeled plane trees on $n+1$ vertices is given by the $n$th Catalan number $C_{n}=\frac{1}{n+1}\left(\begin{array}{c}2 n \\ n\end{array}\right)$ (see [4, ex. 6.19]). Thus we have

$$
\left|\mathcal{P F}_{n}\right|=\left|\mathcal{P}_{n+1, n+1}\right|=n ! \cdot C_{n}=2 n(2 n-1) \cdots(n+2) .
$$

A binary tree is a tree in which each vertex has at most two children and each child of a vertex is designated as its left or right child. We denote by $\mathcal{B}_{n}$ the set of binary trees on $[n]$ and by $\mathcal{B}_{n}^{\text {bi }}$ the set of bicolored binary trees on $[n]$.

For $k \geq 2$, a $k$-ary tree is a tree where each vertex has at most $k$ children and each child of a vertex is designated as its first, second,..., or $k$ th child. We denote by $\mathcal{A}_{n}^{k}$ the set of $k$-ary trees on $[n]$. Clearly, we have that $\mathcal{A}_{n}^{2}=\mathcal{B}_{n}$. Since the number of unlabeled $k$-ary trees on $n$ vertices is given by $\frac{1}{(k-1) n+1}\left(\begin{array}{c}k n \\ n\end{array}\right)$ (see $\left[4\right.$, p. 172]), the cardinality of $\mathcal{A}_{n}^{k}$ is as follows:

$$
\left|\mathcal{A}_{n}^{k}\right|=n ! \cdot \frac{1}{(k-1) n+1}\left(\begin{array}{c}
k n \\
n
\end{array}\right)=k n(k n-1) \cdots(k n-n+2) .
$$

Now we introduce a combinatorial interpretation of the number RHS $_{n}$. Let $\mathfrak{b}$ be an unlabeled binary tree on $n$ vertices and $\omega: V(\mathfrak{b}) \rightarrow[n]$ be a bijection. Then the pair $(\mathfrak{b}, \omega)$ is identified with a (labeled) binary tree on $[n]$. Let $\Pi(\mathfrak{b}, \omega)$ be the set of vertices $v$ in $\mathfrak{b}$ such that $v$ has no descendant $v^{\prime}$ satisfying $\omega(v)>\omega\left(v^{\prime}\right)$, i.e., $\omega(v)$ is proper.

Let $\mathcal{D}_{n}$ be the set of bicolored binary trees on $[n]$ such that each proper vertex is colored with $\mathbf{b}$ or $\mathbf{w}$ and each improper vertex is colored with $\mathbf{b}$.

Lemma 1. The cardinality of $\mathcal{D}_{n}$ is equal to $\mathrm{RHS}_{n}$.

Proof. Let $\mathcal{D}_{n}^{\prime}$ be the set defined as follows:

$$
\mathcal{D}_{n}^{\prime}:=\left\{(\mathfrak{b}, \omega, \alpha) \mid(\mathfrak{b}, \omega) \in \mathcal{B}_{n} \text { and } \alpha \subseteq \Pi(\mathfrak{b}, \omega)\right\}
$$

There is a canonical bijection from $\mathcal{D}_{n}^{\prime}$ to $\mathcal{D}_{n}$ as follows: Given $(\mathfrak{b}, \omega, \alpha) \in \mathcal{D}_{n}^{\prime}$, if a vertex $v$ of $\mathfrak{b}$ is contained in $\alpha$ then color $v$ with $\mathbf{w}$; otherwise color $v$ with $\mathbf{b}$. Thus it suffices to show that the cardinality of $\mathcal{D}_{n}^{\prime}$ equals $\mathrm{RHS}_{n}$.

Given an unlabeled binary tree $\mathfrak{b}$ and a subset $\alpha$ of $V(\mathfrak{b})$, let $l(\mathfrak{b}, \alpha)$ be the number of labelings $\omega$ satisfying $\alpha \subseteq \Pi(\mathfrak{b}, \omega)$. Then for each $v \in \alpha$, the label $\omega(v)$ of $v$ should be the smallest among the labels of the descendants of $v$. If we pick a labeling $\omega$ uniformly at random, the probability that $\omega(v)$ is the smallest among the labels of the descendants of $v$ is $1 / h(v)$. So the number of possible labelings $\omega$ is $n ! / \prod_{v \in \alpha} h(v)$. Thus we have

$$
\begin{aligned}
\left|\mathcal{D}_{n}^{\prime}\right| & =\sum_{\mathfrak{b}} \sum_{\alpha \subseteq V(\mathfrak{b})} l(\mathfrak{b}, \alpha) \\
& =\sum_{\mathfrak{b}} \sum_{\alpha \subseteq V(\mathfrak{b})} n ! \prod_{v \in \alpha} \frac{1}{h(v)}
\end{aligned}
$$

which coincides with $\mathrm{RHS}_{n}$. 


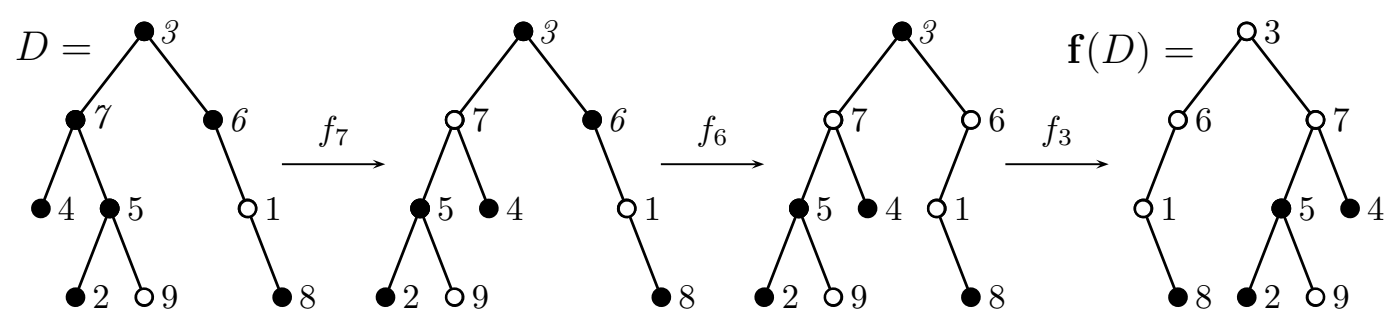

Figure 1: The map f. (Right improper vertices are in italics.)

\section{A bijection}

In this section we construct a bijection between $\mathcal{F}_{n}^{\text {bi }}$ and $\mathcal{D}_{n}$, which gives a bijective proof of $(2)$.

Given a vertex $v$ of a bicolored binary tree $B$, let $L(B, v)$ (resp. $R(B, v)$ ) be the descendant subtree of $B$, which is rooted at the left (resp. right) child of $v$. Note that $L(B, v)$ and $R(B, v)$ may be empty, but $L(B, v)$ or $R(B, v)$ is nonempty when $v$ is improper. For any kind of tree $T$, let $m(T)$ be the smallest vertex in $T$. By convention, we put $m(\emptyset)=\infty$. For an improper vertex $v$ of $B$, if $m(L(B, v))>m(R(B, v))$, then we say that $v$ is right improper; otherwise left improper.

For a vertex $v$ of $B$, define the flip on $v$, which will be denoted by $f_{v}$, by swapping $L(B, v)$ and $R(B, v)$ and changing the color of $v$. Note that the flip satisfies $f_{v} \circ f_{v}=i d$ and $f_{v} \circ f_{w}=f_{w} \circ f_{v}$. For a bicolored binary tree $D$ in $\mathcal{D}_{n}$, let $\mathbf{f}$ be the map defined by

$$
\mathbf{f}(D):=\left(f_{v_{1}} \circ \cdots \circ f_{v_{k}}\right)(D)
$$

where $\left\{v_{1}, \ldots, v_{k}\right\}$ is the set of right improper vertices in $D$. (See Figure 1.)

Let $\mathcal{E}_{n}$ be the set of bicolored binary trees $E$ on $[n]$ such that every improper vertex $v$ is left improper, i.e., $m(L(E, v))<m(R(E, v))$.

Lemma 2. The map $\mathbf{f}$ is a bijection from $\mathcal{D}_{n}$ to $\mathcal{E}_{n}$.

Proof. For a bicolored binary tree $E$ in $\mathcal{E}_{n}$, let $\mathbf{f}^{\prime}$ be the map defined by $\mathbf{f}^{\prime}(E):=\left(f_{u_{1}} \circ\right.$ $\left.\cdots \circ f_{u_{j}}\right)(E)$, where $\left\{u_{1}, \ldots, u_{j}\right\}$ is the set of white-colored improper vertices in $E$. Then the map $\mathbf{f}^{\prime}$ is the inverse of $\mathbf{f}$.

Let $\mathcal{G}_{n}$ (resp. $\mathcal{Q}_{n}$ ) be the set of bicolored trees (resp. bicolored plane trees) on $[n+1]$ such that $n+1$ is the root colored with $\mathbf{b}$. Note that the map $\gamma$ (resp. $\bar{\gamma}$ ) given at the beginning of Section 2 can be regarded as a bijection $\gamma: \mathcal{G}_{n} \rightarrow \mathcal{F}_{n}^{\text {bi }}\left(\right.$ resp. $\bar{\gamma}: \mathcal{Q}_{n} \rightarrow \mathcal{P} \mathcal{F}_{n}^{\text {bi }}$ ). For a vertex $v$ of $Q \in \mathcal{Q}_{n}$, let $\left(w_{1}, \ldots, w_{r}\right)$ be the children of $v$, in order. Then for each $i=1, \ldots, r-1$, we say that $w_{i+1}$ is the right sibling of $w_{i}$. The set $\mathcal{G}_{n}$ can be viewed as a subset of $\mathcal{Q}_{n}$ satisfying the following condition: Suppose that $v$ is the right sibling of $u$ in $Q \in \mathcal{Q}_{n}$. Then $m(S(Q, u))<m(S(Q, v))$ holds.

Recall that $\mathcal{B}_{n}^{\text {bi }}$ denotes the set of bicolored binary trees on $[n]$. Clearly we have $\mathcal{E}_{n} \subseteq$ $\mathcal{B}_{n}^{\text {bi }}$. Let $\Phi$ be a bijection from $\mathcal{B}_{n}^{\text {bi }}$ to $\mathcal{Q}_{n}$, which maps $B$ to $Q$ as follows: 


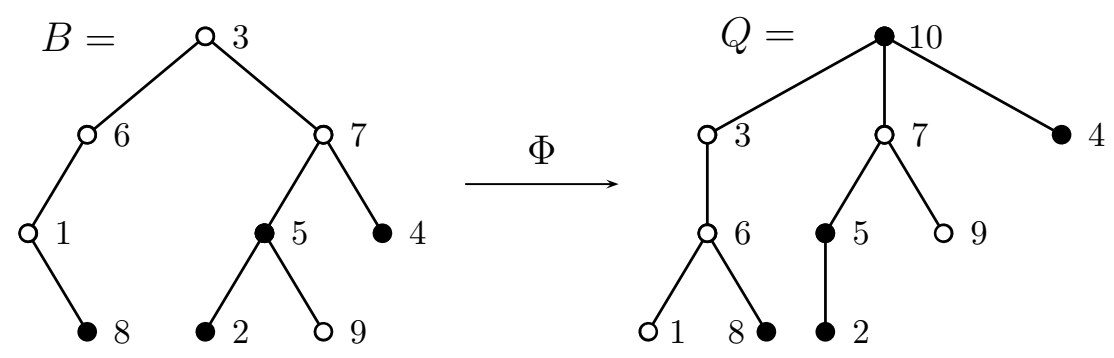

Figure 2: The bijection $\Phi$.

1. The root of $B$ is the first child of $n+1$ in $Q$.

2. $v$ is the first child of $u$ in $Q$ iff $v$ is a left child of $u$ in $B$.

3. $v$ is the right sibling of $u$ in $Q$ iff $v$ is a right child of $u$ in $B$.

4. The color of $v$ in $Q$ is the same as the color of $v$ in $B$.

Note that here $\Phi$ is essentially an extension of a well-known bijection, which is described in $[5$, p. 60], from binary trees to plane trees.

Lemma 3. The restriction $\phi$ of $\Phi$ to $\mathcal{E}_{n}$ is a bijection from $\mathcal{E}_{n}$ to $\mathcal{G}_{n}$.

Proof. For any improper vertex $v$ of $E \in \mathcal{E}_{n}$, we have $m(L(E, v))<m(R(E, v))$. This guarantees that $m(S(G, v))<m(S(G, w))$ in $G=\Phi(E)$, where $w$ (if it exists) is the right sibling of $v$ in $G$. Thus $\Phi(E) \in \mathcal{G}_{n}$, i.e., $\Phi\left(\mathcal{E}_{n}\right) \subseteq \mathcal{G}_{n}$. Similarly we can show that $\Phi^{-1}\left(\mathcal{G}_{n}\right) \subseteq \mathcal{E}_{n}$. So we have $\Phi\left(\mathcal{E}_{n}\right)=\mathcal{G}_{n}$, which implies $\phi$ is bijective.

From Lemma 3 , we easily get that $\gamma \circ \phi$ is a bijection from $\mathcal{E}_{n}$ to $\mathcal{F}_{n}^{\text {bi }}$. Combining this result with Lemma 2 yields the following consequence.

Theorem 4. The map $\gamma \circ \phi \circ \mathbf{f}$ is a bijection from $\mathcal{D}_{n}$ to $\mathcal{F}_{n}^{\text {bi }}$.

Figure 3 shows how the bijection in Theorem 4 maps a bicolored binary tree $D$ in $\mathcal{D}_{11}$ to a bicolored forest $F$ on [11]. From equation (3) the cardinality of $\mathcal{F}_{n}^{\text {bi }}$ equals $\mathrm{LHS}_{n}$ and from Lemma 1 the cardinality of $\mathcal{D}_{n}$ equals $\mathrm{RHS}_{n}$. Thus Theorem 4 is a combinatorial explanation of identity (2).

\section{Generalized formulas}

Theorem 4 implies the set $\mathcal{D}_{n}$ of binary trees on $[n]$ such that each proper vertex is colored with the color $\mathbf{b}$ or $\mathbf{w}$ and each improper vertex is colored with the color $\mathbf{b}$ has cardinality $\left|\mathcal{D}_{n}\right|=2^{n}(n+1)^{n-1}$. In this section we give a generalization of this result. 

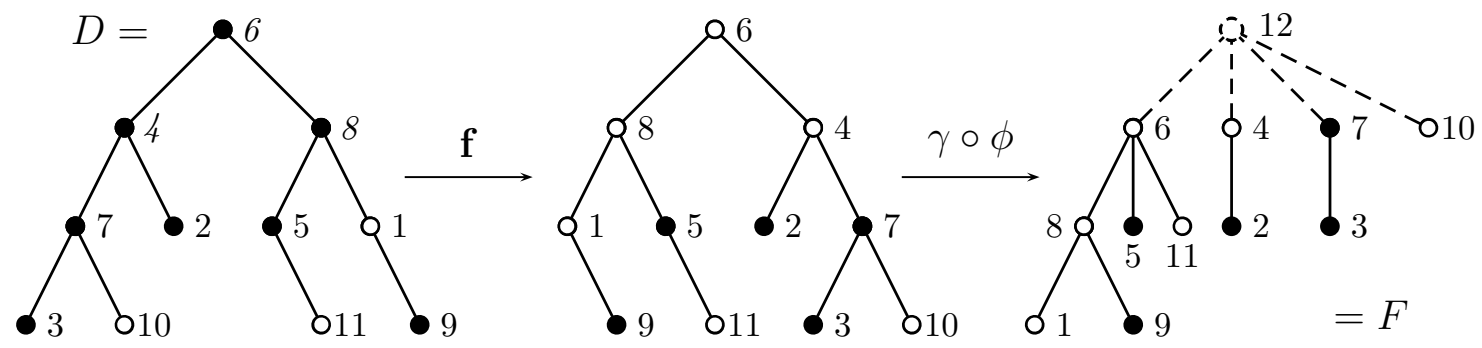

Figure 3: The bijection from $\mathcal{D}_{n}$ to $\mathcal{F}_{n}^{\text {bi }}$.

For $n \geq 1$, let $a_{n, m}$ denote the number of $k$-ary trees on $[n]$ with $m$ proper vertices. By convention, we put $a_{0, m}=\delta_{0, m}$. Let

$$
a_{n}(t)=\sum_{m \geq 0} a_{n, m} t^{m}=\sum_{T \in \mathcal{A}_{n}^{k}} t^{\mathrm{pv}(T)},
$$

where $\operatorname{pv}(T)$ is the number of proper vertices of $T$. It is clear that for a positive integer $t$ the number $a_{n}(t)$ is the number of $k$-ary trees on $[n]$ such that each proper vertex is colored with the color $\overline{1}, \overline{2}, \ldots$, or $\bar{t}$ and each improper vertex has one color $\overline{1}$. Let $A(x)$ be denote the exponential generating function for $a_{n}(t)$, i.e.,

$$
A(x)=\sum_{n \geq 0} a_{n}(t) \frac{x^{n}}{n !} .
$$

Lemma 5. The generating function $A=A(x)$ satisfies the following differential equation:

$$
A^{\prime}=k x A^{k-1} A^{\prime}+t A^{k},
$$

where the prime denotes the derivative with respect to $x$.

Proof. Let $T$ be an $k$-ary tree on $[n] \cup\{0\}$. Delete all edges going from the root $r$ of $T$. Then $T$ is decomposed into $T^{\prime}=\left(r ; T_{1}, \ldots, T_{k}\right)$ where each $T_{i}$ is a $k$-ary tree and $[n] \cup\{0\}$ is the disjoint union of $V\left(T_{1}\right), \ldots, V\left(T_{k}\right)$ and $\{r\}$. Consider two cases: (i) For some $1 \leq i \leq k, T_{i}$ has the vertex 0 ; (ii) $r=0$. Then we have

$$
\begin{aligned}
a_{n+1}(t)= & \sum_{i=1}^{k} \sum_{n_{1}+\cdots+n_{k}=n-1}\left(\begin{array}{c}
n \\
1, n_{1}, \ldots, n_{k}
\end{array}\right) a_{n_{1}}(t) \cdots a_{n_{i}+1}(t) \cdots a_{n_{k}}(t) \\
& +t \sum_{n_{1}+\cdots+n_{k}=n}\left(\begin{array}{c}
n \\
n_{1}, \ldots, n_{k}
\end{array}\right) a_{n_{1}}(t) \cdots a_{n_{k}}(t) .
\end{aligned}
$$

Multiplying both sides by $x^{n} / n$ ! and summing over $n$ yields (5).

To compute $a_{n}(t)$ from (5) we need the following theorem. 
Theorem 6. Fix positive integers $a$ and $b$. Let $u=1+\sum_{n=1}^{\infty} u_{n} x^{n} / n$ ! be a formal power series in $x$ satisfying

$$
u^{\prime}=a x u^{b} u^{\prime}+t u^{b+1}
$$

Then $u_{n}$ is given by

$$
u_{n}=t \prod_{i=1}^{n-1}((b i+1) t+a(n-i)), \quad n \geq 1
$$

Proof. Adding $(b t-a) x u^{b} u^{\prime}$ to both sides of (6) yields

$$
\left(1+(b t-a) x u^{b}\right) u^{\prime}=t\left(b x u^{b-1} u^{\prime}+u^{b}\right) u .
$$

Since $\left(1+(b t-a) x u^{b}\right)^{\prime}=(b t-a)\left(b x u^{b-1} u^{\prime}+u^{b}\right)$, we have

$$
(b t-a) \log u=t \log \left(1+(b t-a) x u^{b}\right) .
$$

Taking the exponential of both sides and the substitutions $x=y^{b}$ and $y u\left(y^{b}\right)=\hat{u}(y)$ yield

$$
\hat{u}(y)=y\left(1+(b t-a) \hat{u}(y)^{b}\right)^{t /(b t-a)} .
$$

Applying the Lagrange Inversion Formula (see [4, p. 38]) to (7) yields that

$$
\begin{aligned}
{\left[y^{b n+1}\right] \hat{u}(y) } & =\frac{1}{b n+1}\left[y^{b n}\right]\left(1+(b t-a) y^{b}\right)^{\frac{t(b n+1)}{b t-a}} \\
& =\frac{1}{b n+1}(b t-a)^{n}\left(\begin{array}{c}
\frac{t(b n+1)}{b t-a} \\
n
\end{array}\right) \\
& =\frac{t}{n !} \prod_{i=1}^{n-1}(t(b n+1)-(b t-a) i)
\end{aligned}
$$

Since $u_{n}=n !\left[y^{b n+1}\right] \hat{u}(y)$, we obtain the desired result.

Since (5) is a special case of (6) $(a=k, b=k-1)$, we can deduce a formula for $a_{n}(t)$. Corollary 7 ( $k$-ary trees). For $n \geq 1, a_{n}(t)$ is given by

$$
a_{n}(t)=t \prod_{i=1}^{n-1}((k i-i+1) t+k(n-i)) .
$$

Clearly, substituting $t=1$ in (8) yields the number of $k$-ary trees on $[n]$, i.e.,

$$
a_{n}(1)=k n(k n-1) \cdots(k n-n+2)=\left|\mathcal{A}_{n}^{k}\right| .
$$


For some values of $k$, we can get interesting results. In particular when $k=2$ we have

$$
a_{n}(t)=t \prod_{i=1}^{n-1}((i+1) t+2(n-i)) \stackrel{t=2}{\longrightarrow} 2^{n}(n+1)^{n-1},
$$

so this is a generalization of $\left|\mathcal{D}_{n}\right|=2^{n}(n+1)^{n-1}$, i.e., identity (2).

In fact Theorem 6 has more applications. For $n \geq 1$, let $f_{n, m}$ denote the number of forests on $[n]$ with $m$ proper vertices and let $p_{n, m}$ denote the number of plane forests on $[n]$ with $m$ proper vertices. Let

$$
f_{n}(t)=\sum_{m \geq 1} f_{n, m} t^{m} \quad \text { and } \quad p_{n}(t)=\sum_{m \geq 1} p_{n, m} t^{m} .
$$

Let $F(x)$ and $P(x)$ be the exponential generating function for $f_{n}(t)$ and $p_{n}(t)$, respectively, i.e.,

$$
F(x)=1+\sum_{n \geq 1} f_{n}(t) \frac{x^{n}}{n !} \quad \text { and } \quad P(x)=1+\sum_{n \geq 1} p_{n}(t) \frac{x^{n}}{n !} .
$$

Similarly to Lemma 5, we can get two differential equations:

$$
\begin{aligned}
& F^{\prime}=x F F^{\prime}+t F^{2}, \\
& P^{\prime}=x P^{2} P^{\prime}+t P^{3} .
\end{aligned}
$$

Since (9) and (10) are special cases of (6) $(a=b=1$ and $a=1, b=2$, respectively), we have the following results.

Corollary 8. Suppose $f_{n}(t)$ and $p_{n}(t)$ are defined as above. Then we have

1. For $n \geq 1, f_{n}(t)$ is given by

$$
f_{n}(t)=t \prod_{i=1}^{n-1}((i+1) t+(n-i)) .
$$

2. For $n \geq 1, p_{n}(t)$ is given by

$$
p_{n}(t)=t \prod_{i=1}^{n-1}((2 i+1) t+(n-i)) .
$$

Note that (11) and (12) are generalizations of (3) and (4), respectively. Moreover, from these formulas, we can easily get

$$
\begin{aligned}
& \sum_{T \in \mathcal{T}_{n+1}} t^{\mathrm{pv}(T)}=t \prod_{i=0}^{n-1}((i+1) t+(n-i)), \\
& \sum_{P \in \mathcal{P}_{n+1}} t^{\mathrm{pv}(P)}=t \prod_{i=0}^{n-1}((2 i+1) t+(n-i)),
\end{aligned}
$$

which are generalizations of $\left|\mathcal{T}_{n+1}\right|=(n+1)^{n}$ and $\left|\mathcal{P}_{n+1}\right|=(n+1)$ ! $C_{n}$.

Remark. In spite of the simple expressions, we have not proved (8), (11) and (12) in a bijective way. Also a direct combinatorial proof of Theorem 6 would be desirable. 


\section{Acknowledgment}

The author thanks Ira Gessel for his helpful advice and encouragement. The author also thanks the referees for useful comments and suggestions.

\section{References}

[1] A. Cayley, A theorem on trees, Quart. J. Math. 23 (1889), 376-378.

[2] J. Pitman and R. P. Stanley, A polytope related to empirical distributions, plane trees, parking functions, and the associahedron, Discrete Comput. Geom. 27 (2002), no. 4, 603-634.

[3] A. Postnikov, Permutohedra, associahedra, and beyond, Retrospective in Combinatorics: Honoring Richard Stanley's 60th Birthday, Massachusetts Institute of Technology, Cambridge, Massachusetts, June 22-26, 2004, slide available at: http://www-math.mit.edu/ apost/talks/perm-slides.pdf

[4] R. P. Stanley, Enumerative Combinatorics, Vol. 2, Cambridge University Press, Cambridge, 1999.

[5] D. Stanton and D. White, Constructive Combinatorics, Springer-Verlag, New York, 1986. 